\title{
The Effect on the Immune System in the Human Body Due to COVID-19: An Insight on Traditional to Modern Approach as a Preventive Measure
}

\author{
Jugal Sutradhar ${ }^{1 *}$, Bapi Ray Sarkar ${ }^{2}$ \\ ${ }^{1}$ Harishchandra PG College, Institute of Pharmacy, Bawan Beegha, Varanasi, India \\ ${ }^{2}$ Department of Pharmaceutical Technology, University of North Bengal (NBU), Raja Rammohunpur, Darjeeling, West Bengal, India
}

Received November 22, 2020

Reviewed August 17, 2021

Accepted November 26, 2021

\section{*Corresponding Author} Jugal Sutradhar

Harishchandra PG College, Institute of Pharmacy, Bawan Beegha, Varanasi

221002, India

Tel: +91-938-933-6032

E-mail: jugalsutradhar@gmail.com
The COVID-19, the most infectious pandemic disease arising due to SARS-CoV-2 (Severe Acute Respiratory Syndrome Coronavirus 2) has caused huge issues globally. In this review, we discuss the impact of COVID-19 on the immune system of the human body and the protective mechanisms of the host immune system opposing viral infections. Here, we summarize the effect of the pandemic of the novel coronavirus disease on the immune system such as sleep and Behavioral Immune System (BIS) together with consideration of researcher's observation points of view. We draw particular attention to recent up-to-date reports concerning COVID-19 drugs as well as information about the landscape document for COVID-19 vaccines released by WHO (World Health Organization), and some adverse events of COVID-19 vaccination. Additionally, can take part in the preventive appraise in opposition within this pandemic severe COVID-19 infections disease may affect some outcome in physical exercise, physical movement, healthy diets, and good nutrition are significant for supporting the immune systems and summarize AYUSH (Ayurveda, Yoga and Naturopathy, Unani, Siddha, and Homeopathy) Indian medicinal systems guidelines for immunity boosting procedures during COVID-19 pandemic.

Keywords: COVID-19, immunity, sleep, behavioral immune system, immunity-boosting procedures

\section{INTRODUCTION}

Coronavirus Disease (COVID-19), a global pandemic, and today's most infectious disease is caused by a novel severe acute respiratory syndrome coronavirus 2 (SARS-CoV-2) [1]. This new coronavirus was found in Wuhan City, China, [2] and rapidly spread globally [3]. The Middle East Respiratory Syndrome coronavirus (MERS-CoV) along with severe acute respiratory syndrome coronavirus (SARS-CoV) both causative agents are related to the COVID-19 stains [4]. The infection is spread from human to human [5] and through contacting infected droplets released into the surroundings by coughing and sneezing, as well as contact spread through the mouth, nasal, and ocular mucous [6]. For COVID-19 infection, common symptoms include fever, dry cough, tiredness, breathing difficulty, body pain, chills, sore throat, headache, weight loss, gastric disturbance, loss of smell and taste, skin rashes, and discoloration of toes and fingers $[7,8]$.

Tyrell and Bynoe first narrated coronavirus in 1966 from a common cold patient. Coronavirus is a sphere-shaped virion among a core-shell and solar corona sticks out on the surface; according to the Latin word, the meaning of corona is crown [9]. Coronaviruses (family- Coronaviridae) are single-stranded with positive-strand ribonucleic acid (RNA) [10]. There are twenty-eight identical species among four genera such as $\alpha$ (alpha), $\beta$ (beta), $\gamma$ (gamma), and $\delta$ (delta) coronaviruses recently classified by the ICTV (International Committee on Taxonomy of Viruses) [11], and among these $\beta$ coronaviruses are responsible for novel severe acute respiratory syndrome coronavirus 2 (SARS-CoV-2) [12]. The viral genome size is between $27 \mathrm{~kb}$ to 
$32 \mathrm{~kb}$ and encodes structural as well as non-structural proteins. The structural proteins include the nucleocapsid protein, envelope protein, membrane glycoprotein, and spike protein $[9,13]$. The infection process of SARS-CoV-2 is initiated by the binding of the ACE-2 (angiotensin-converting enzyme 2) receptor on the outer surface membrane. The virus slowly fuses the host cell and harms their original physiological activity [14] (Fig. 1). Severe Acute Respiratory Syndrome Coronavirus (SARS-CoV) shows less severe human infection while SARS-CoV-2 is easily transmitted [15].

The immune system protects against many infectious diseases including COVID-19 [16]. The human body's immune system can defend against various types of diseases, maintain physical condition, protect against harmful effects of substances, and fight germs. The innate immunity is a protection mechanism (primary line), which is related to the host immune system. The innate immune system's impacts can theoretically play an important role to prevent COVID-19 viral disease [17, 18]. The World Health Organization (WHO) suggests for people during this COVID-19 pandemic to maintain regular physical activity such as physical exercise and physical movement [19]. A healthy diet and good nutrition support the immune systems as well as reduce the possibility of developing various types of diseases, and according to the Indian medicinal system AYUSH, some ayurvedic measures and self-care immunityboosting procedures can improve immunity and reduce the probability of getting the COVID-19 disease [20, 21]. The purpose of this review article may be helpful to the development of the immune system of the human being along with reducing the risk of the COVID-19 warrior's before safe effective approved drugs as well as vaccinations and provided information useful to researchers.

\section{EFFECTS OF THE IMMUNE SYSTEM}

\section{The immune system in the human body}

Most micro-organisms live on the body surface, the intestine, the skin, and the respiratory tract [22]. The human body's organs in respect to the immune system can (i) protect against disease; (ii) maintain physical condition and pathogeneses; and (iii) fight germs, reduce the harmful effect of the substance, and prevent abnormal new growth of disorganized tissue. White blood cells (WBC) in blood vessels play an important role in the immune system as they monitor for attacking microbes, and exchange cells in the body and fluids connecting with blood along with lymphatic vessels. Lymphatic vessels (every lymph node) have a specific compartment and can encounter antigens. Immune cells along with external particles move into the lymph node by the lymphatic vessels and finally, these are transported to tissues all over the body and the cycle is continuing. Immune cells are accumulated and distributed with antigens within the lymph nodes as well as spleens compartments [17].

\section{The innate immune system}

The innate immune system is a primary line of protection in opposition to viral infections. Activation of the innate immune response is an early step of the adaptive immune response. Then the host immune system receives the pathogen utilizing PRRs (pattern recognition receptors) and these mostly recognize the viral nucleic acids such as double-stranded RNA
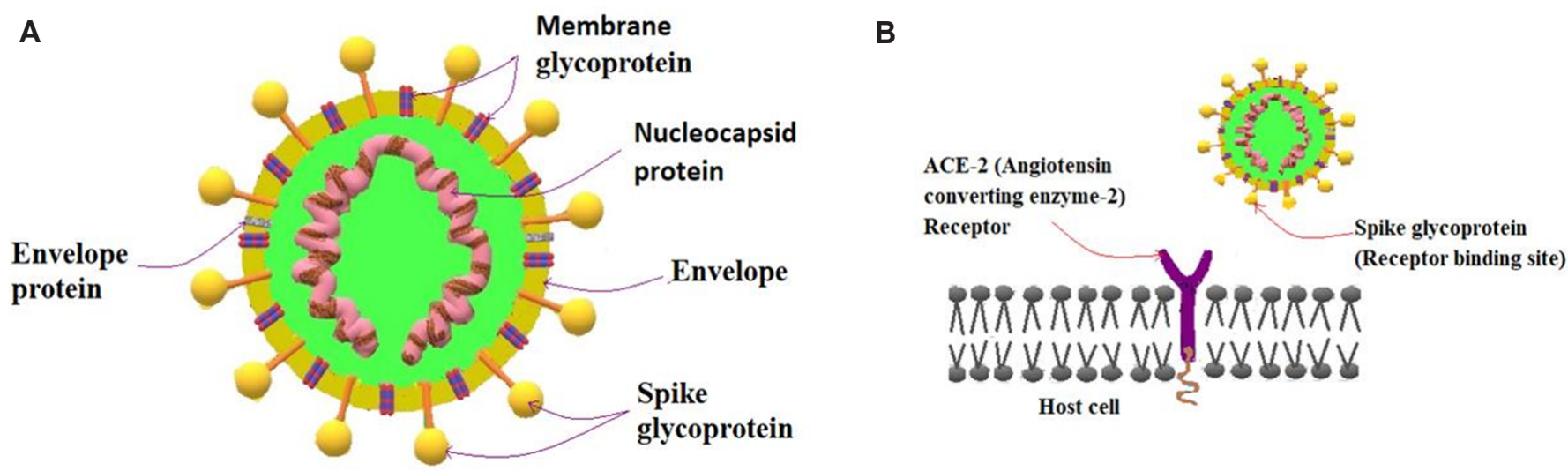

Figure 1. (A) Schematic diagram of SARS-CoV-2 and (B) The schematic diagram shows the spike glycoprotein binds to the receptor ACE-2 (angiotensin-converting enzyme 2). 
(ribonucleic acid), genomic DNA (deoxyribonucleic acid), and single-stranded RNA. The human disease effect a result is recognized by the RNA viruses like COVID-19 disease, Ebola disease, Influenza, HTLV-1 (Lymphotropic virus type 1), and polio measles. This recognition stimulates immune-mediated factors such as IFN (Interferon type-I) along with inflammatory cytokines which cause the antiviral effects $[18,23]$. The cytokines are an important component of the inflammatory process and are produced by several immune cells including dendritic cells (DCs), innate macrophages, natural killer cells (NK cells), adaptive B lymphocytes (B cells), and T lymphocytes ( $\mathrm{T}$ cells) [24]. Removing the regulation of the innate immune response is theoretically plays a key role in the destructive or protective responses to the deadly COVID-19 [18].

\section{Two phases of the immune response initiated by COVID-19 disease}

Immune response in response to COVID-19 infections involves the following two phases [1]. The first phase is described by the immune protection based and the immunity exacerbation phase [25]. Having an internal cause (endogenous) defensive immune response during its period of incubation and in the non-severe stage, a healthy person with human leukocyte antigen (HLA) should provoke an antiviral immune response [26].

The second phase is represented by wide inflammation and immunity suppression [25]. A cytokine storm (CS) describes macrophage activation syndrome (MSA) or secondary hemophagocytic lymphohistiocytosis (sHLH) at the severe stage leading to further referring to tissues along with organ damage $[1,27]$.

\section{COVID-19 PANDEMIC EFFECTS ON THE IMMUNE SYSTEM}

\section{Sleep quality and the immune system}

Targa et al. [28] found that based on the Pittsburgh Sleep Quality Index (PSQI), for the duration of COVID-19 sleep quality was reduced. Reduced sleep quality is connected to negative mood, depression, and anger. To support a healthy life and improve the immune system, sleep is most important.

Descriptive arrangements of the relationship are linking to the immune response, airway infections, shift work, sleeps balance, and COVID-19 disease. The virus gives rise to stress responses brings about a signature of inflammatory markers that important for airway infections. Shift workers are more deprived or have poor sleep and have a higher occurrence of common airway infections. Also, they have a breakdown in the immune response to COVID-19 disease compared to regular schedule workers. A lack of sleep induces airway infection of viruses caused by immune response breakdown. Infected cells of shift workers or sleep deprivation people may not produce heat shock protein 90 (HSP90) and heat shock protein 70 (HSP70) efficiently. These proteins may possibly impact airway infection and anti-viral protection by decreasing dorsal column spinal cord stimulation (DCS), argon plasma coagulation (APC), natural killer cells (NK cells), Toll-like receptors (TLRs), major histocompatibility complex (MHC), interleukin (IL), chemokine (C-C motif) ligand (CCL), tumor necrosis factor alpha (TNF- $\alpha$ ), interferon-gamma (IFN $\gamma$ ), vascular endothelial growth factor (VEGF), adhesion molecule expression (CD+), T-helper lymphocytes 1/2 (Th1/Th2), Toll-like receptor 2/4 (TLR2/TLR4), antigen-presenting cells (APCs), heat shock factor (HSF), and T/B lymphocytes (LT/LB) [29]. The activities of NK cells as well as cytokines, specifically IL-I (Interleukin-I), are connected to sleep. In the sleep-wake process, IL-I with additional immunological peptides, temperature (thermal systems), and immune neuroendocrines are involved. Disturbance of the sleep-wake system can manifest in a disease-like infection [30]. In this current pandemic situation, circadian rhythm sleep-wake disorders (CRSWD) have been found in a group of people. Disruption of the circadian rhythmicity and the immune system may enhance the infection [31].

\section{The behavioral immune system}

The behavioral immune system (BIS) is a supplemental set over the mechanism that works to first-line protection against pathogens and develops preventive behavior [32, 33]. The BIS consists of three essential psychological mechanisms, which include be fond of becoming receptive to the presence concerning infectious pathogens within the instantaneous atmosphere, activate disease-related emotions as well as cognitive responses, and smoothing the progress of behavioral prevention with the action indicated by the pathogen infection [3]. The behavioral immune system leads by persuasion to the adaptive affective, cognitive, and behavioral responses to advance disease prevention [32]. 
Healthcare and essential workers during this COVID-19 pandemic face infection risks and increased stress. The newly developed COVID stress scales (CSS) include five categories of stressors (a) economic and social stress, (b) contamination and danger fear, (c) traumatic stress symptoms, (d) xenophobia, and (e) behavior of reassurance and checking [34].

McKay et al. [35] investigated and explored the idea that the provider's behavior along with their reaction would be associated with their viewpoint regarding exposure therapy. This includes behavioral immune system (BIS) activation, which would be connected to the negative attitudes in the direction of delivery of revelation and explore healthcare provider working attitudes to revelation. Based on the analyzed data, they have examined the role of the behavioral immune system and the pandemic stress (COVID-19) during attitudes to exposure for contamination related fears similar to the obsessive-compulsive disorder (OCD), like avoiding different places and surfaces of public restrooms, doorknobs, and also washing groceries and their containers.

\section{UP-TO-DATE INFORMATION ON COVID-19 DRUGS AND VACCINATION}

\section{The latest information on drugs}

The World Health Organization (WHO) released its solidarity trial results on the $15^{\text {th }}$ of October 2020. After examining overall mortality, start of ventilation, and hospitalized patients in over thirty countries, drugs such as Hydroxychloroquine, Remdesivir, Interferon regimens, and Lopinavir or Ritonavir showed little or no impact on the twenty-eight-day mortality of hospitalized COVID-19 patients. Now, WHO is evaluating the use of immunomodulators, newer Antiviral drugs, and antiSARS CoV-2 monoclonal antibodies [36] (Table 1).

Table 1. Latest COVID-19 drugs response by WHO

\begin{tabular}{lc}
\hline \multicolumn{1}{c}{ Drugs } & Action against COVID-19 \\
\hline Hydroxychloroquine & Less or no effect \\
Remdesivir & \\
Interferon regimens & \\
Lopinavir or Ritonavir & Under evaluation \\
Immunomodulators & \\
Newer antiviral drugs & \\
Anti-SARS CoV-2 monoclonal antibodies & \\
\hline
\end{tabular}

\section{Latest information on vaccination}

WHO released a landscape document for COVID-19 vaccines, which gave information on the novel coronavirus vaccine data. The WHO will not approve any product (vaccine) without verification of the accuracy. The WHO released this draft on the $19^{\text {th }}$ of October (Publication 'Draft landscape of COVID-19 candidate vaccines' $419.8 \mathrm{kB}, 11$ pages PDF), which contained 44 candidate vaccines undergoing clinical evaluation considering the vaccine developer/manufacturers (e.g. Sinovac and University of Oxford/AstraZeneca), vaccine platform (e.g. inactivated and non-replicating viral vector), type of candidate vaccine (e.g. inactivated and ChAdOx1-S), number of doses (e.g. 1 or 2), the timing of doses (e.g. 0,14 days and 0, 21 days), route of administration (e.g. IM and ID), 'clinical stage' which contains three phases (Phase 1, Phase $1 / 2$, Phase 2, and Phase 3 ), and the update information site with reference to the direction of the clinical stages [e.g. for Sinovac Vaccine available site of Phase 1/2 (NCT04383574; NCT04352608; NCT04551547) along with Phase 3 available (NCT04456595; 669/UN6.KEP/ EC/2020; NCT04582344)] [37].

\section{Adverse events of COVID-19 vaccination}

The whole world is moving toward vaccine development for the specific SARS-CoV-2 antigen. The mandatory data is insufficient for this vaccine development because (i) the $S$ protein in the virus is given good ground for expecting antigen, on the other hand, best or most effective use design a most favorable immune response and some debates like targeted to the receptor-binding area otherwise full length of the protein, (ii) the severe acute respiratory syndrome (SARS), as well as the Middle East respiratory syndrome (MERS) vaccine development, resulted in exacerbated lung disease and also adverse type 2 helper $\mathrm{T}$ cell responses, (iii) single-dose vaccines will be and the possible period of immunity is unknown. From a safety point of view vaccine development is a lengthy process [8].

\section{COMPLEMENTARY, ALTERNATIVE, AND}

TRADITIONAL APPROACHES TO THE BOOST IMMUNE SYSTEM

\section{General measure}

In this COVID-19 pandemic, the WHO suggests that people 
regularly maintain physical exercise and physical movement which can help to improve blood circulation and muscle activity. This is also beneficial for the mind and reduces the risk of depression. Regular exercise reduces high blood pressure and decreases the risk of stroke, heart disease, type- 2 diabetes, and various types of cancers. Lack of exercise increases susceptibility to COVID-19 disease [19].

Throughout the COVID-19 pandemic, a healthy diet is extremely important to prevent infection and recover or recuperate from the infection. However, no dietary supplement or food can cure or prevent COVID-19 infection. Healthy diets and good nutrition are important for supporting the immune system and minimizing the probability of developing various health problems like heart disease, obesity, diabetes, and cancer. The WHO suggests a healthy diet during this pandemic such as (i) eating a variety of foods including vegetables, fruits, whole grains (rice, wheat, and maize), animal protein (milk, eggs, fish, and meat), legumes (beans \& lentils), wholegrain foods (unprocessed oats, maize, millet, and brown rice), and snacks (raw vegetables, unsalted nuts, and fresh fruit). (ii) Reduce salt intake. (iii) Limit intake of sweets and sugar. (iv) Eat a moderate quantity of fats (butter, lard, and ghee) substances, and oils (olive, sunflower, and soy). (v) Drink sufficient water. (vi) Avoid drinking alcohol which reduces protection against COVID-19.

(vii) Breastfeed as breast milk provides nutrients and contains antibodies that protect infants or babies [21].

\section{Ayurvedic immunity improving measures}

Referred to as AYUSH (Ayurveda, yoga and naturopathy, unani, siddha, and homeopathy) Indian medicinal system has recommended self-care immunity-boosting procedures for preventive measures during the COVID-19 pandemic. The guidelines are as follows, (i) general Measures: drink warm water daily, daily thirty minutes practice Pranayama, Yogasana along with meditation. In cooking use spices such as turmeric, cumin, and garlic. (ii) Ayurvedic immunity improving measures: in the morning take Chyavanprash (sugar-free Chyavanprash for diabetics). Drink tea (herbal) with basil, cinnamon, black pepper, dry Ginger, and raisin one or two times per day. Drink turmeric milk (one hundred fifty milliliters hot milk with half teaspoon turmeric powder) one or two times per day. (iii) Simple Ayurvedic measures: in both nostrils apply coconut oil or sesame oil or ghee two times a day. Gurgle warm water with coconut oil or sesame oil (one tablespoon) one or two times per day. (iv) If you have a sore throat or dry cough: steam inhalation practiced with fresh mint leaves or caraway seeds once a day. For a cough clove powder with honey or natural sugar two to three times a day. These eminent are collected from throughout the country and may improve immunity [20, 38] (Fig. 2).

Preventive appraise in opposition to COVID-19 pandemic

Drink worm water daily

Daily thirty minutes practice Pranayama Yogasana along with meditation

In cooking used spices such as Turmeric, Cumin and Garlic

General Measures

In both nostrils apply coconut oil or sesame oil or ghee two times a day

Gurgle one or two times per day by warm water with coconut oil or sesame oil (one table spoon)
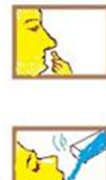

Drink turmeric milk one or two times per day

Drink tea (herbal) with Basil, Cinnamon Black pepper, Dry Ginger and Raisin per day one or two times

In morning time take Chyavanprash and take sugar free Chyavanprash for diabetics person

\section{Ayurvedic Immunity improving Measures}

Steam inhalation practiced with fresh Mint leaves or Caraway seeds once time in a day

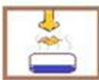

In cough otherwise in throat irritation Clove powder with honey or natural sugar two to three times a day

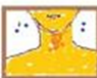

Figure 2. Immunity boosting procedures for the duration of COVID-19 pandemic. 


\section{DISCUSSION}

The immune system is the primary protection against many infectious diseases. A specific adaptive immune response in non-severe stages and the incubation period is required to remove the virus and to prevent the disease expansion [16]. Sufficient numbers of different types of vitamins, minerals, and several nutrient substances help activate the immune system [39]. To protect against viral infection it is important to balance the nutrition, according to Calder et al. [40]. Healthy eating habits with adequate nutritional balance and maintenance, immuneboosting measurement, or an active lifestyle could decrease the load of the COVID-19 pandemic $[41,42]$. A study (71 participants) from Targa et al. [28] showed that sleep quality was reduced during the COVID-19 pandemic. McKay et al. [35] have investigated BIS activation during this pandemic and the attitude which was related to fear leading to OCD. Similarly, some preventive measures such as physical exercise, healthy diets, physical movement, good nutrition, and sleep are significant for assisting the immune systems, as well as boosting the immune system. The COVID-19 pandemic affects the immune system such as sleep and the behavioral immune system. During this COVID-19 pandemic crisis, some self-care immunity-boosting procedures such as general measures, Ayurvedic immunity improving measures, sore throat, or dry cough measurement, and simple Ayurvedic measures can fight against viral infection. Physical activity and a healthy diet (eating a variety of food, vegetables, and fruits, reducing salt intake, limiting the intake of sweets and sugar, eating moderate quantity of fats, avoiding drinking alcohol, and drinking sufficient water) can reduce the probability as well as the severity of COVID-19 infection.

According to WHO solidarity trial results, previously used drugs have shown little or no effect [36]. Every affected country is trying to find possible long-term solutions in the form of safe and effective drugs and vaccines. In this review, we focus on disease prevention with alternative medicines as well as newer medicines including vaccines. This review does not provide any information on curing the disease, but these findings will help researchers and people to accumulate the knowledge to overcome the pandemic. Until now, limited studies have been conducted on this new COVID-19 viral disease and lots of information is still unknown, thus further studies will be helpful. Future studies should determine more characteristics of this viral disease and examine various types of immune-boosting measures with correlation studies including huge numbers of human volunteers.

\section{CONCLUSIONS}

At present, approximately every part of the world is working hard to discover an accurate and safe treatment or vaccination for COVID-19. Until now there is no approved treatment or vaccination for COVID-19 disease. During this pandemic, prevention and self-conscious strategies may reduce the risk of infection. Also, some immunotherapy can fight against viral infection. In this review, we concluded that the immune system impacts sleep and the behavioral immune system. We conclude that lifestyle adjustment (such as pranayama or yoga asana along with meditation), nutritional management, simple Ayurvedic measures, and preventive interference can improve the human immune system and are helpful during the COVID-19 crisis. This review provides new ideas for the prevention against COVID-19 related-conditions by boosting immunity power as well as other preventive procedures. We believe that this paper will help as a stimulus for other researchers and give new hope to the people with regards to their wellbeing.

\section{DECLARATION}

The authors declare that all authors have contributed to the paper and have never submitted the manuscript, in whole or in part, to other journals.

\section{CONFLICT OF INTEREST}

The authors declare that there is no conflict of interest.

\section{ORCID}

Jugal Sutradhar, https://orcid.org/0000-0003-2369-5178

Bapi Ray Sarkar, https://orcid.org/0000-0001-6513-8543

\section{REFERENCES}

1. Jesenak M, Brndiarova M, Urbancikova I, Rennerova Z, Vojtkova J, Bobcakova A, et al. Immune parameters and COVID-19 infection - associations with clinical severity and disease prognosis. Front Cell Infect Microbiol. 2020;10:364.

2. Prompetchara E, Ketloy C, Palaga T. Immune responses in COVID-19 and potential vaccines: lessons learned from SARS and 
MERS epidemic. Asian Pac J Allergy Immunol. 2020;38(1):1-9.

3. Panahi L, Amiri M, Pouy S. Risks of novel coronavirus disease (COVID-19) in pregnancy; a narrative review. Arch Acad Emerg Med. 2020;8(1):e34.

4. Kannan S, Shaik Syed Ali P, Sheeza A, Hemalatha K. COVID-19 (novel coronavirus 2019) - recent trends. Eur Rev Med Pharmacol Sci. 2020;24(4):2006-11.

5. Pascarella G, Strumia A, Piliego C, Bruno F, Del Buono R, Costa F, et al. COVID-19 diagnosis and management: a comprehensive review. J Intern Med. 2020;288(2):192-206.

6. Esakandari H, Nabi-Afjadi M, Fakkari-Afjadi J, Farahmandian N, Miresmaeili SM, Bahreini E. A comprehensive review of COVID-19 characteristics. Biol Proced Online. 2020;22:19.

7. Kaur SP, Gupta V. COVID-19 vaccine: a comprehensive status report. Virus Res. 2020;288:198114.

8. Bhagat S, Yadav N, Shah J, Dave H, Swaraj S, Tripathi S, et al. Novel corona virus (COVID-19) pandemic: current status and possible strategies for detection and treatment of the disease. Expert Rev Anti Infect Ther. 2020. doi: 10.1080/14787210.2021. 1835469. [Epub ahead of print]

9. Velavan TP, Meyer CG. The COVID-19 epidemic. Trop Med Int Health. 2020;25(3):278-80.

10. Ye Q, Wang B, Mao J. The pathogenesis and treatment of the 'Cytokine Storm' in COVID-19. J Infect. 2020;80(6):607-13.

11. Naserghandi A, Allameh SF, Saffarpour R. All about COVID-19 in brief. New Microbes New Infect. 2020;35:100678.

12. Harapan H, Itoh N, Yufika A, Winardi W, Keam S, Te H, et al. Coronavirus disease 2019 (COVID-19): a literature review. J Infect Public Health. 2020;13(5):667-73.

13. Shanmugaraj B, Siriwattananon K, Wangkanont K, Phoolcharoen W. Perspectives on monoclonal antibody therapy as potential therapeutic intervention for Coronavirus disease-19 (COVID-19). Asian Pac J Allergy Immunol. 2020;38(1):10-8.

14. Wan DY, Luo XY, Dong W, Zhang ZW. Current practice and potential strategy in diagnosing COVID-19. Eur Rev Med Pharmacol Sci. 2020;24(8):4548-53.

15. Guo YR, Cao QD, Hong ZS, Tan YY, Chen SD, Jin HJ, et al. The origin, transmission and clinical therapies on coronavirus disease 2019 (COVID-19) outbreak - an update on the status. Mil Med Res. 2020;7(1):11.

16. Nilashi M, Samad S, Yusuf SYM, Akbari E. Can complementary and alternative medicines be beneficial in the treatment of COVID-19 through improving immune system function? J Infect Public Health. 2020;13(6):893-6.

17. Chowdhury MA, Hossain N, Kashem MA, Shahid MA, Alam A. Immune response in COVID-19: a review. J Infect Public Health. 2020;13(11):1619-29.

18. Kim KD, Hwang I, Ku KB, Lee S, Kim SJ, Kim C. Progress and challenges in the development of COVID-19 vaccines and current understanding of SARS-CoV-2- specific immune responses. J Microbiol Biotechnol. 2020;30(8):1109-15.

19. WHO. HealthyAtHome - Physical Activity [Internet]. Geneva: WHO; c2021 [cited 2020 Oct 23]. Available from: https://www. who.int/news-room/campaigns/connecting-the-world-tocombat-coronavirus/healthyathome/healthyathome---physicalactivity.

20. Ministry of Ayush. Home: Ayurveda's Immunity Boosting Measures for Self Care During COVID 19 Crisis [Internet]. New Delhi: Ministry of Ayush; [cited 2020 Oct 26]. Available from: https://www.ayush.gov.in/.

21. WHO. HealthyAtHome - Healthy Diet [Internet]. Geneva: WHO; c2021 [cited 2020 Oct 23]. Available from: https://www. who.int/campaigns/connecting-the-world-to-combat-coronavirus/healthyathome/healthyathome---healthy-diet.

22. Duerkop BA, Hooper LV. Resident viruses and their interactions with the immune system. Nat Immunol. 2013;14(7):654-9.

23. Poltronieri P, Sun B, Mallardo M. RNA viruses: RNA roles in pathogenesis, coreplication and viral load. Curr Genomics. 2015;16(5):327-35.

24. Ragab D, Salah Eldin H, Taeimah M, Khattab R, Salem R. The COVID-19 cytokine storm; what we know so far. Front Immunol. 2020;11:1446.

25. Paces J, Strizova Z, Smrz D, Cerny J. COVID-19 and the immune system. Physiol Res. 2020;69(3):379-88.

26. Shi Y, Wang Y, Shao C, Huang J, Gan J, Huang X, et al. COVID-19 infection: the perspectives on immune responses. Cell Death Differ. 2020;27(5):1451-4.

27. Tufan A, Avanoğlu Güler A, Matucci-Cerinic M. COVID-19, immune system response, hyperinflammation and repurposing antirheumatic drugs. Turk J Med Sci. 2020;50(SI-1):620-32.

28. Targa ADS, Benítez ID, Moncusí-Moix A, Arguimbau M, de Batlle J, Dalmases M, et al. Decrease in sleep quality during COVID-19 outbreak. Sleep Breath. 2021;25(2):1055-61.

29. Silva FRD, Guerreiro RC, Andrade HA, Stieler E, Silva A, de Mello MT. Does the compromised sleep and circadian disruption of night and shiftworkers make them highly vulnerable to 2019 coronavirus disease (COVID-19)? Chronobiol Int. 2020;37(5):607-17.

30. Moldofsky H. Sleep and the immune system. Int J Immunopharmacol. 1995;17(8):649-54.

31. Bryson WJ. Circadian rhythm sleep-wake disorders and the COVID-19 pandemic. J Clin Sleep Med. 2020;16(8):1423.

32. Terrizzi JA, Shook NJ, McDaniel MA. The behavioral immune system and social conservatism: a meta-analysis. Evol Hum Behav. 2013;34(2):99-108.

33. Schaller M, Park JH. The behavioral immune system (and why it 
matters). Curr Dir Psychol Sci. 2011;20(2):99-103.

34. McKay D, Asmundson GJG. Substance use and abuse associated with the behavioral immune system during COVID-19: the special case of healthcare workers and essential workers. Addict Behav. 2020;110:106522.

35. McKay D, Minaya C, Storch EA. Conducting exposure and response prevention treatment for contamination fears during COVID-19: the behavioral immune system impact on clinician approaches to treatment. J Anxiety Disord. 2020;74:102270.

36. WHO. News Release for COVID-19 [Internet]. Geneva: WHO; [cited 2020 Oct 20]. Available from: https://www.who.int/news/ item/15-10-2020-solidarity-therapeutics-trial-produces-conclusive-evidence-on-the-effectiveness-of-repurposed-drugs-forcovid-19-in-record-time.

37. WHO. Draft Landscape of COVID-19 Candidate Vaccines [Internet]. Geneva: WHO; [cited 2020 Oct 22]. Available from: https:/www.who.int/publications/m/item/draft-landscape-ofcovid-19-candidate-vaccines.
38. Tillu G, Chaturvedi S, Chopra A, Patwardhan B. Public health approach of Ayurveda and Yoga for COVID-19 prophylaxis. J Altern Complement Med. 2020;26(5):360-4.

39. Zildzic M, Masic I, Salihefendic N, Jasic M, Hajdarevic B. The importance of nutrition in boosting immunity for prevention and treatment COVID-19. Int J Biomed Healthc. 2020;8(2):739.

40. Calder PC, Carr AC, Gombart AF, Eggersdorfer M. Optimal nutritional status for a well-functioning immune system is an important factor to protect against viral infections. Nutrients. 2020;12(4):1181.

41. Di Renzo L, Gualtieri P, Pivari F, Soldati L, Attinà A, Cinelli G, et al. Eating habits and lifestyle changes during COVID-19 lockdown: an Italian survey. J Transl Med. 2020;18(1):229.

42. Esobi IC, Lasode MK, Barriguete MOF. The impact of COVID-19 on healthy eating habits. J Clin Nutr Heal. 2020;1(1):0012. 